\title{
COSMIC MICROWAVE BACKGROUND POLARIZATION AND TEMPERATURE POWER SPECTRA ESTIMATION USING LINEAR COMBINATION OF WMAP 5 YEAR MAPS
}

\author{
Pramoda Kumar Samal ${ }^{1}$, Rajib Saha ${ }^{2,3}$, Jacques Delabrouille ${ }^{4}$, Simon Prunet ${ }^{5}$, Pankaj Jain ${ }^{1}$, and Tarun SouradeeP ${ }^{6}$ \\ ${ }^{1}$ Department of Physics, Indian Institute of Technology, Kanpur, India \\ 2 Jet Propulsion Laboratory, M/S 169-327, 4800 Oak Grove Drive, Pasadena, CA 91109, USA \\ ${ }^{3}$ California Institute of Technology, Pasadena, CA 91125 , USA \\ ${ }^{4}$ CNRS, Laboratoire APC, 10, rue Alice Domon et Léonie Duquet, 75205 Paris, France \\ ${ }_{5}$ Institut d'Astrophysique de Paris, 98 bis Boulevard Arago, F-75014 Paris, France \\ ${ }^{6}$ Inter-University Centre for Astronomy and Astrophysics, Post Bag 4, Ganeshkhind, Pune 411007, India \\ Received 2009 March 26; accepted 2010 March 2; published 2010 April 15
}

\begin{abstract}
We estimate cosmic microwave background (CMB) polarization and temperature power spectra using Wilkinson Microwave Anisotropy Probe (WMAP) 5 year foreground contaminated maps. The power spectrum is estimated by using a model-independent method, which does not utilize directly the diffuse foreground templates nor the detector noise model. The method essentially consists of two steps: (1) removal of diffuse foregrounds contamination by making linear combination of individual maps in harmonic space and (2) cross-correlation of foreground cleaned maps to minimize detector noise bias. For the temperature power spectrum we also estimate and subtract residual unresolved point source contamination in the cross-power spectrum using the point source model provided by the $W M A P$ science team. Our $T T, T E$, and $E E$ power spectra are in good agreement with the published results of the WMAP science team. We perform detailed numerical simulations to test for bias in our procedure. We find that the bias is small in almost all cases. A negative bias at low $l$ in $T T$ power spectrum has been pointed out in an earlier publication. We find that the bias-corrected quadrupole power $\left(l(l+1) C_{l} / 2 \pi\right)$ is $532 \mu \mathrm{K}^{2}$, approximately 2.5 times the estimate $\left(213.4 \mu \mathrm{K}^{2}\right)$ made by the WMAP team.
\end{abstract}

Key words: cosmic background radiation - methods: data analysis - polarization

Online-only material: color figures

\section{INTRODUCTION}

The anisotropies of the cosmic microwave background $(\mathrm{CMB})$ radiation are the most important evidence behind the tiny fluctuations that are generated by the inflationary paradigm of the big bang cosmology (Starobinsky 1982; Guth \& Pi 1982; Bardeen et al. 1983). One can determine cosmological parameters precisely by measuring these anisotropies (Jungman et al. 1996a, 1996b; Bond et al. 1997; Zaldarriaga et al. 1997). These anisotropies possess a certain degree of linear polarization due to the quadrupolar temperature pattern seen by the moving electrons in the primordial plasma (Rees 1968; Basko \& Polnarev 1980). Recently, the Wilkinson Microwave Anisotropy Probe $(W M A P)$ satellite has mapped the total intensity and polarization of the CMB anisotropies over the full sky in its five frequency bands from $23 \mathrm{GHz}$ to $94 \mathrm{GHz}$ with unprecedented resolution and sensitivity (Bennett et al. 2003a, 2003b; Page et al. 2007; Kogut et al. 2007; Hinshaw et al. 2009). The polarization power spectrum acts as a complement to the temperature power spectrum. It leads to better constraints on the cosmological parameters and is also useful to break degeneracies among certain cosmological parameters, e.g., epoch of re-ionization and scalarto-tensor ratio (Kinney 1998). Furthermore, it has been argued that CMB polarization may serve as a direct probe of inflation (Spergel \& Zaldarriaga 1997), can test if the parity symmetry is preserved on the cosmological scales (Lue et al. 1999; Komatsu et al. 2009), can provide information about the epoch when the first stars begin to form (Crittenden et al. 1993; $\mathrm{Ng} \& \mathrm{Ng}$ 1995), and provide a measure of the gravity waves that are generated by inflation (Harari \& Zaldarriaga 1993; Crittenden et al. 1995; Kamionkowski \& Kosowsky 1998). The WMAP team has pro- duced their temperature and polarization power spectrum based upon the foreground cleaned maps which are obtained using prior models of the synchrotron, dust, and free-free components (Kogut et al. 2007; Page et al. 2007). Though this method allows one to use all the available information about the foreground components it is also a very important scientific task to perform an independent analysis of the data by techniques which do not rely upon explicit foreground modeling.

A multipole based approach for foreground removal was first proposed by Tegmark \& Efstathiou (1996) and was implemented on the WMAP data by Tegmark et al. (2003). Later, Saha et al. (2006, 2008) and Souradeep et al. (2006) extended this method to extract the temperature anisotropy power spectrum of the CMB radiation from the raw WMAP data. The power spectrum is obtained by forming several cleaned maps using subsets of the available maps and thereafter cross-correlating the resulting maps. This internal power spectrum estimation (IPSE) method utilizes CMB data as the only input without making any explicit modeling of the diffuse galactic foreground components or detector noise bias. The foreground components are removed using the fact that in thermodynamic temperature unit, the $\mathrm{CMB}$ signal is predicted to be independent of frequency since it follows a blackbody spectrum (Mather et al. 1994; Fixsen et al. 1996), while the foreground components are frequency dependent. The detector noise bias is removed by cross-correlating different foreground cleaned maps obtained by using independent detector subsets. This substantially removes the noise bias since, to a good approximation, WMAP detector noise is uncorrelated for two different detectors (Jarosik et al. 2003, 2007). The final power spectra (Saha et al. 2006, 2008) obtained by IPSE agree well with the results published by the WMAP 
science team. Thus, the method serves as an independent technique to verify the main power spectrum result obtained by the WMAP science team starting from the stage of diffuse foreground components removal. The method has several advantages. First, the foreground components removal method is entirely independent of the foreground template models. Therefore, the foreground cleaned maps are not susceptible to systematic errors that might arise in template based methods due to incorrect template modeling. Second, the cleaned power spectrum can be studied analytically in the special case of full-sky one iteration foreground cleaning (Saha et al. 2008). This allows us to quantify and understand the statistical properties of the residuals in the cleaned power spectrum. This may be very useful in the case of noisy data or when the total number of available frequency bands are less than the total number of independent parameters required for satisfactory modeling of all dominant underlying components. A detailed analytical study of the bias in the cleaned power spectrum is presented in Saha et al. (2008). Third, it is possible to obtain a model independent estimate of the map and power spectrum of the total foreground emission at each of the frequency bands (Ghosh et al. 2009).

In this paper, we extract CMB polarization $E E, T E$, as well as the $T T$ power spectra using the WMAP 5 year foreground and detector noise contaminated maps as input to IPSE-our combined foreground removal and power spectrum estimation procedure. Since the CMB polarization signal is weak, the polarized maps published by the WMAP science team are dominated by the foreground components and detector noise. This seriously limits the accuracy with which the polarized CMB power spectrum can be extracted. However, since our method does not use any template model to remove foreground components we argue that our power spectrum is free from systematic effects that might arise due to incorrect modeling of polarized (and temperature) foreground templates.

The error bars as well as the bias in the extracted polarization power spectrum are estimated by numerical Monte Carlo simulations. Here we make use of explicit foreground and detector noise models. In the case of temperature power spectrum a similar analysis reveals the presence of a negative bias at low multipoles (Saha et al. 2008). The bias-corrected temperature spectra explain almost all of the low power observed in case of quadrupole. In this case, it is also possible to analytically obtain an estimate of the bias in some special cases.

Alternate approaches of CMB power spectrum estimation have been studied by several authors, e.g., using foreground cleaned maps provided by the WMAP science team (Fosalba \& Szapudi 2004; Patanchon et al. 2005; Eriksen et al. 2007a, 2007b), as well as using uncleaned maps where some models of foregrounds and (or) detector noise are necessary (Eriksen et al. 2008a, 2008b). Other approaches for foreground cleaning, using needlet coefficients (Delabrouille et al. 2009) and harmonic variance minimization (Kim et al. 2008, 2009), have also been proposed.

The organization of our paper is as follows. We describe the methodology of power spectrum estimation in Section 2. We present the power spectrum results along with their covariance structure in Section 3. Finally, we conclude in Section 4.

\section{METHOD}

The basic procedure for extracting the temperature power spectrum is described in Saha et al. (2006, 2008). Here we generalize this to include polarization. The basic maps for the case of polarization are available in terms of the Stokes parameters $Q$ and $U$. Since these are coordinate-dependent quantities it is more convenient to work with the coordinate independent $E$ and $B$ modes (Zaldarriaga \& Seljak 1997; Seljak \& Zaldarriaga 1997; Kamionkowski et al. 1997). Another problem with using $Q$ and $U$ maps is that the $E$ and $B$ modes mix with one another when one applies a sky mask (Jaffe et al. 2000; Tegmark \& de Oliveira-Costa 2001; Bunn et al. 2003; Smith \& Zaldarriaga 2007) to remove heavily contaminated Galactic regions. This demands an extra data processing step to isolate the actual CMB $E$ and $B$ mode power spectra from their mixture. To avoid this problem we start by converting fullsky $Q$ and $U$ maps to full sky $E$ and $B$ maps and apply mask whenever required on the resultant maps. This is similar to what is proposed in Betoule et al. (2009) for estimating $r=T / S$ for the Planck satellite mission and the Experimental Probe of Inflationary Cosmology.

We note that conversion of $Q, U$ maps to $E$ maps results in a redistribution of total sky power from lower galactic latitude to higher galactic latitude and vice versa. Although it is convenient to express CMB power in terms of $E, B$ mode power spectrum and it is also possible to transform detector noise covariance matrix from $Q, U$ basis to $E, B$ basis, the polarized foreground components are often modeled in $Q, U$ basis. This is because from the theoretical point of view it is easier to predict the polarized components in $Q, U$ basis starting from the first principles. It has been pointed out by Gold et al. (2009) that the polarized components are sensitive to line-of-sight effect toward galactic plane. This leads to different spectral behavior of polarized and un-polarized components in this region. Redistribution of polarized foreground power due to $Q, U$ to $E$, $B$ conversion would mix the spectral properties and amplitudes of low and high latitude foregrounds. The mixing may cause some additional variation of spectral indices of polarized $E, B$ components in higher galactic latitudes resulting in effectively "additional" polarized components (Bouchet et al. 1995). Despite this leakage of power into higher galactic latitudes, our foreground removal procedure should be applicable even if we work directly with the $E, B$ field maps. While studying statistical properties of the polarized IPSE power spectrum one is required to take into account any "excess" foreground components in higher latitudes resulting from the conversion from $Q, U$ to $E, B$ basis. An interesting study in the future would be to model foreground components in terms of $E, B$ basis instead of $Q, U$ basis.

To obtain the $E$ and $B$ maps we first expand the full sky spin \pm 2 fields $(Q \pm i U)$ in terms of spin-2 spherical harmonics ${ }_{ \pm 2} Y_{l m}(\hat{n})$

$$
\begin{gathered}
(Q+i U)(\hat{n})=\sum_{l m} a_{2, l m}{ }_{2} Y_{l m}(\hat{n}) \\
(Q-i U)(\hat{n})=\sum_{l m} a_{-2, l m-2} Y_{l m}(\hat{n}) .
\end{gathered}
$$

Since both $Q$ and $U$ are real, one can show that the expansion coefficients obey $a_{-2, l m}^{*}=a_{2, l-m}$. The spin- $0 E$ and $B$ are now obtained by the usual spherical harmonic transform,

$$
\begin{aligned}
& E(\hat{n})=\sum_{l \geqslant 2,|m| \leqslant l} a_{l m}^{E} Y_{l m}(\hat{n}) \\
& B(\hat{n})=\sum_{l \geqslant 2,|m| \leqslant l} a_{l m}^{B} Y_{l m}(\hat{n})
\end{aligned}
$$


where

$$
\begin{gathered}
a_{l m}^{E}=\frac{1}{2}\left(a_{2, l m}+a_{-2, l m}\right) \\
a_{l m}^{B}=\frac{1}{2 i}\left(a_{2, l m}-a_{-2, l m}\right) .
\end{gathered}
$$

This gives us ten different full sky maps for each of the $E$ and $B$ fields corresponding to the ten WMAP Differencing Assemblies (DAs). The ten DAs are labeled as $K, K a, Q 1, Q 2, V 1, V 2$, $W 1, W 2, W 3, W 4$ corresponding to the five different frequency channels $K, K a, Q, V$, and $W$. We note that the bands $Q, V$, and $W$ have 2, 2, and 4 DAs, respectively.

The WMAP team does not include the $W$ band in their polarization analysis since it is found to be contaminated by some unknown systematic effects at low $l$. However, as discussed in Nolta et al. (2009), the problem may be caused by poor statistics rather than by a systematic effect. In our analysis we include $W$ band for the entire multipole range. Although by including $W$ band we might introduce some systematic effect in the power spectrum at very small $l$, any effect of such systematics would be small after averaging the power spectrum over a sufficiently large range of multipoles. Furthermore, as supported by our results, inclusion of $W$ band leads to significantly smaller error bars since it allows us to use more data. Nevertheless, we cautiously add that, if one is primarily interested in cosmological parameter estimation using only very low $l E E$ multipoles, a better approach would be to remove the $W$ band. In our present work, we ignore this complication and include the $W$ band in our analysis.

We first eliminate the highly contaminated Galactic plane from all the ten $E$-mode DA maps using to P06 mask (Page et al. 2007). This procedure is slightly different from what was used earlier in order to extract the temperature power spectrum (Saha et al. 2006, 2008). In the latter case, the authors cleaned the entire unmasked sky in nine iterations and also produced full-sky foreground cleaned temperature maps. However, to eliminate potential residual foreground contamination arising from the Galactic plane the temperature mask provided by WMAP is applied before computing the power spectrum. In the present work, to extract the polarization power spectrum, we apply the P06 mask right at the beginning. This has the advantage of being much faster since one can perform the cleaning in a single iteration. However, this does not allow us to produce a full sky polarization map. In order to cross-check our one iteration method we also divide the $E E$ maps in several parts and then perform foreground removal in the iterative approach. We find that the final power spectrum of this method is similar to the one-iteration case.

We select different possible linear combinations of four maps out of the available DAs as described below. The entire set of linear combinations is listed in Table 1. Each of these linear combinations independently leads to a clean map.

The cleaning is accomplished independently for each $l$, by linearly combining these maps with weights, $\hat{w}_{l}^{a}$, such that the spherical harmonic components of the cleaned map are given by

$$
a_{l m}^{\text {Clean }}=\sum_{a=1}^{n_{c}} \hat{w}_{l}^{a} \frac{a_{l m}^{a}}{B_{l}^{a}} .
$$

Here $n_{c}$ is the total number of maps used for cleaning. In the present case of four channel cleaning, $n_{c}=4$. The factor $B_{l}^{a}$ is the circularized beam transform function for the frequency band $a$ (Hill et al. 2009). The weights $\hat{w}_{l}^{a}$ are chosen so as to
Table 1

List of the Different Combinations of the DA Maps, used to Obtain the Final 48 Cleaned Maps, Denoted by $C \mathbf{i}$ and $C A \mathbf{i}$ where $\mathbf{i}=1,2, \ldots, 24$

\begin{tabular}{ll}
\hline \hline$K+Q 1+V 1+W 12=C 1$ & $K a+Q 1+V 1+W 12=\mathrm{CA} 1$ \\
$K+Q 1+V 1+W 13=C 2$ & $K a+Q 1+V 1+W 13=\mathrm{CA} 2$ \\
$K+Q 1+V 1+W 14=C 3$ & $K a+Q 1+V 1+W 14=\mathrm{CA} 3$ \\
$K+Q 1+V 1+W 23=C 4$ & $K a+Q 1+V 1+W 23=\mathrm{CA} 4$ \\
$K+Q 1+V 1+W 24=C 5$ & $K a+Q 1+V 1+W 34=\mathrm{CA} 6$ \\
$K+Q 1+V 1+W 34=C 6$ & $K a+Q 2+V 2+W 12=\mathrm{CA} 7$ \\
$K+Q 2+V 2+W 12=C 7$ & $K a+Q 2+V 2+W 13=\mathrm{CA} 8$ \\
$K+Q 2+V 2+W 13=C 8$ & $K a+Q 2+V 2+W 14=\mathrm{CA} 9$ \\
$K+Q 2+V 2+W 14=C 9$ & $K a+Q 2+V 2+W 23=\mathrm{CA} 10$ \\
$K+Q 2+V 2+W 23=C 10$ & $K a+Q 2+V 2+W 24=\mathrm{CA} 11$ \\
$K+Q 2+V 2+W 24=C 11$ & $K a+Q 2+V 2+W 34=\mathrm{CA} 12$ \\
$K+Q 2+V 2+W 34=C 12$ & $K a+Q 1+V 2+W 12=\mathrm{CA} 13$ \\
$K+Q 1+V 2+W 12=C 13$ & $K a+Q 1+V 2+W 13=\mathrm{CA} 14$ \\
$K+Q 1+V 2+W 13=C 14$ & $K a+Q 1+V 2+W 14=\mathrm{CA} 15$ \\
$K+Q 1+V 2+W 14=C 15$ & $K a+Q 1+V 2+W 23=\mathrm{CA} 16$ \\
$K+Q 1+V 2+W 23=C 16$ & $K a+Q 1+V 2+W 24=\mathrm{CA} 17$ \\
$K+Q 1+V 2+W 24=C 17$ & $K a+Q 1+V 2+W 34=\mathrm{CA} 18$ \\
$K+Q 1+V 2+W 34=C 18$ & $K a+Q 2+V 1+W 12=\mathrm{CA} 19$ \\
$K+Q 2+V 1+W 12=C 19$ & $K a+Q 2+V 1+W 13=\mathrm{CA} 20$ \\
$K+Q 2+V 1+W 13=C 20$ & $K a+Q 2+V 1+W 14=\mathrm{CA} 21$ \\
$K+Q 2+V 1+W 14=C 21$ & $K a+Q 2+V 1+W 23=\mathrm{CA} 22$ \\
$K+Q 2+V 1+W 23=C 22$ & $K a+Q 2+V 1+W 24=\mathrm{CA} 23$ \\
$K+Q 2+V 1+W 24=C 23$ & $K a+Q 2+V 1+W 34=\mathrm{CA} 24$ \\
$K+Q 2+V 1+W 34=C 24$ &
\end{tabular}

minimize the total power subject to the constraint

$$
\hat{\mathbf{W}}_{l} \mathbf{e}_{0}=\mathbf{e}_{0}^{T} \hat{\mathbf{W}}_{l}^{T}=1
$$

where $\mathbf{e}_{0}$ is a column vector with unit elements

$$
\mathbf{e}_{0}=\left(\begin{array}{c}
1 \\
\ldots \\
\cdots \\
1
\end{array}\right)
$$

and $\hat{\mathbf{W}}_{l}$ is the row vector $\left(\hat{w}_{l}^{1}, \hat{w}_{l}^{2}, \ldots, \hat{w}_{l}^{n_{c}}\right)$. This constraint is required so as to preserve the $\mathrm{CMB}$ signal. The weights are obtained using the empirical covariance matrix, $\hat{\mathbf{C}}_{l}$, by the relationship (Saha et al. 2006, 2008; Tegmark et al. 2003; Tegmark \& Efstathiou 1996; Eriksen et al. 2004; Delabrouille \& Cardoso 2009)

$$
\hat{\mathbf{W}}_{l}=\frac{\mathbf{e}_{0}^{T}\left(\hat{\mathbf{C}}_{l}\right)^{-1}}{\mathbf{e}_{0}^{T}\left(\hat{\mathbf{C}}_{l}\right)^{-1} \mathbf{e}_{0}}
$$

We label the resulting cleaned maps as $\mathrm{Ci}$ and CAi where $\mathbf{i}=1,2, \ldots, 24$. Here the maps Ci use the DAs $K$ along with possible combinations of DAs from the bands $Q, V$, and $W$. Similarly, the maps CAi include $K a$ instead of $K$. The entire nomenclature is listed in Table 1 . In the case of the $W$ band we average over two DAs before we start the foreground cleaning. Hence in Table 1 the notation W12, for example, refers to the average of the DAs $W 1$ and $W 2$. This averaging is not essential to the procedure and one may also directly use the original WMAP DAs. However, averaging leads to a reduced detector noise in each cleaned map.

After obtaining the 48 cleaned maps we cross-correlate them in selected combinations in order to reduce the contribution due to detector noise. We cross-correlate all pairs of maps such that the two cleaned maps in each pair are formed by distinct DAs. 
Table 2

List of All the 24 Cross-power Spectra using the 48 Cleaned Maps, C1, C2, ., C24 and CA1, CA2, .., CA24

\begin{tabular}{|c|c|c|c|c|c|}
\hline $\mathrm{C} 1 \otimes \mathrm{CA} 12$ & $\mathrm{C} 2 \otimes \mathrm{CA} 11$ & $\mathrm{C} 3 \otimes \mathrm{CA} 10$ & $\mathrm{C} 4 \otimes \mathrm{CA} 9$ & $\mathrm{C} 5 \otimes \mathrm{CA} 8$ & $\mathrm{C} 6 \otimes \mathrm{CA} 7$ \\
\hline $\mathrm{C} 7 \otimes \mathrm{CA} 6$ & $\mathrm{C} 8 \otimes \mathrm{CA} 5$ & $\mathrm{C} 9 \otimes \mathrm{CA} 4$ & $\mathrm{C} 10 \otimes \mathrm{CA} 3$ & $\mathrm{C} 11 \otimes \mathrm{CA} 2$ & $\mathrm{C} 12 \otimes \mathrm{CA} 1$ \\
\hline $\mathrm{C} 13 \otimes \mathrm{CA} 24$ & $\mathrm{C} 14 \otimes \mathrm{CA} 23$ & $\mathrm{C} 15 \otimes \mathrm{CA} 22$ & $\mathrm{C} 16 \otimes \mathrm{CA} 21$ & $\mathrm{C} 17 \otimes \mathrm{CA} 20$ & $\mathrm{C} 18 \otimes \mathrm{CA} 19$ \\
\hline $\mathrm{C} 19 \otimes \mathrm{CA} 18$ & $\mathrm{C} 20 \otimes \mathrm{CA} 17$ & $\mathrm{C} 21 \otimes \mathrm{CA} 16$ & $\mathrm{C} 22 \otimes \mathrm{CA} 15$ & $\mathrm{C} 23 \otimes \mathrm{CA} 14$ & $\mathrm{C} 24 \otimes \mathrm{CA} 13$ \\
\hline
\end{tabular}

This gives us 24 cross-correlated power spectra on the masked sky. All the possible cross-correlations are listed in Table 2.

We convert each of the 24 masked sky power spectra into full sky estimates of the underlying CMB power spectrum using the mode-mode coupling matrix corresponding to the P06 mask following the MASTER approach (Hivon et al. 2002; Hinshaw et al. 2003; Tristram et al. 2005). We then remove beam and pixel effects from each of these 24 full-sky power spectra. Our final $E E$ power spectrum is simply a uniform average of these 24 cross-spectra. We rely upon Monte Carlo simulations to compute the error bars as well as possible bias in the extracted power spectrum.

The neighboring multipoles in the power spectrum become coupled since the spherical harmonics lose orthogonality on a masked sky. Hence, to obtain full information about the twopoint correlation function of the resulting power spectrum one needs to construct the covariance matrix,

$$
\left\langle\Delta C_{l} \Delta C_{l^{\prime}}\right\rangle=\left\langle\left(C_{l}-\left\langle C_{l}\right\rangle\right)\left(C_{l^{\prime}}-\left\langle C_{l^{\prime}}\right\rangle\right)\right\rangle .
$$

We compute the covariance matrix by Monte Carlo simulations. The correlations can be minimized by suitably binning the power spectrum. We use a binning identical to that used by the WMAP team. Let $C_{\alpha}$ denote the binned power spectrum. Then the covariance matrix of the binned spectrum is obtained as

$$
\left\langle\Delta C_{\alpha} \Delta C_{\alpha^{\prime}}\right\rangle=\left\langle\left(C_{\alpha}-\left\langle C_{\alpha}\right\rangle\right)\left(C_{\alpha^{\prime}}-\left\langle C_{\alpha^{\prime}}\right\rangle\right)\right\rangle .
$$

The standard deviation obtained from the diagonal elements of the binned covariance matrix gives the error bars on the binned final spectrum. Since the cosmic variance of the CMB power spectrum decays as $\sim 1 /(2 l+1)$, the diagonal terms in the above correlation matrix decay with increasing multipoles. For a visual comparison of correlation between different bins we define a correlation matrix, $C_{\alpha \alpha^{\prime}}$, of the binned power spectrum, where

$$
C_{\alpha \alpha^{\prime}}=\frac{\left\langle\Delta C_{\alpha} \Delta C_{\alpha^{\prime}}\right\rangle}{\sqrt{\left\langle\left(\Delta C_{\alpha}\right)^{2}\right\rangle\left\langle\left(\Delta C_{\alpha^{\prime}}\right)^{2}\right\rangle}} .
$$

All the elements of this matrix are bound to lie between $[-1,1]$.

\subsection{Bias Analysis}

The IPSE method, described above, contains some bias in the estimation of the CMB power spectrum. This is not just a problem with the IPSE technique. All methods used to extract power spectrum, including the template fitting method, contain a certain level of bias. In the case of the template fitting method, one cannot rule out the presence of some systematic error in the foreground model. This will lead to bias in the estimated power spectrum. It is, therefore, important to make a reliable estimate of bias in all techniques used for extraction of CMB power spectrum. The bias in IPSE can be studied analytically in some special cases (Saha et al. 2008) if we consider the full sky power spectrum. These analytic results have so far not been generalized for the case of partial sky power spectrum, which is required in practice. In this work, we estimate and remove this bias by performing Monte Carlo simulations. These simulations assume certain models for $\mathrm{CMB}$, foregrounds, and detector noise. It is clear that if this bias turns out to be very large then some of the attractiveness of the IPSE method is lost. For the temperature power spectrum the bias was found to be relatively small for the entire multipole range, except at $l=2$ (Saha et al. 2008). At $l=2$ one finds a significant negative bias. This negative bias also has a very interesting analytical explanation which we discuss below. Furthermore this negative bias provides a very simple explanation for the low power extracted both by IPSE and the WMAP team at $l=2$. In this paper, we find that the bias is small in almost all the multiple bins for the polarization power spectrum also. A significant bias is found only for the lowest multipole bin for the case of the EE power spectrum. However, we note that such a bias is not to be considered as a limiting factor of the IPSE method, since the WMAP experiment was not optimized for the CMB polarization observation. The problem of $\mathrm{CMB}$ extraction turns out to be very complicated especially when the noise in the data becomes strong.

In Saha et al. (2008), the analytic discussion of bias was confined to the case where the power spectrum is obtained by using auto-correlations of the different cleaned maps. This is not applicable directly to the IPSE method which uses crosscorrelations. However, qualitatively the residual foregrounds and CMB bias for auto-correlation and cross-correlation would be very similar. The main advantage of cross-correlation is the absence of detector noise bias.

The analytical bias results of IPSE power spectrum provide measures of residuals at each multipole. In their regime of applicability these results allow one to unambiguously perform bias correction on each scale. Since it is expected that different sources of bias, e.g., detector noise and foregrounds, operate on different angular scales, the analytical results are also very useful to identify easily different sources of bias at different multipole regions.

An interesting feature of the IPSE power spectrum is the existence of a negative bias at the lowest multipoles. The negative bias arises because of a chance correlation of the largest scale $\mathrm{CMB}$ modes with other non-CMB components on the given realization of our universe. However, the negative bias rapidly decreases with $l$. For an IPSE power spectrum estimated from the entire sky the negative bias is given by $-c \frac{C_{l}^{c}}{2 l+1}$, where $C_{l}^{c}$ is the theoretical CMB power spectrum. The value of the factor, $c$, depends upon the total number of foreground components or the number of available frequency bands. If the detector noise is negligible on the largest scales and total number of components happen to be less than or equal to the available frequency bands the factor $c$ is given by $c=n_{f}$, where $n_{f}$ is the number of foreground components. If detector noise cannot be ignored even on the largest scale or the total number of components exceeds the number of frequency bands $(n)$, then we have $c=(n-1)$.

We next discuss the bias as a function of the multipole $l$. If the detector noise is negligible and one has more frequency bands than the number of foreground components the IPSE power 
spectrum does not contain any bias other than the possible negative bias which decreases rapidly with multipoles. The magnitude of the foreground components does not play a crucial role in this case. In principle, the IPSE method would work perfectly in this case. Considering the intermediate multipole region, if the detector noise is still negligible, the IPSE power spectrum would be free from any bias provided $n_{f}<n$. If one increases the number of foregrounds such that $n_{f} \geqslant n$ a positive foreground bias becomes visible in the power spectrum, even if the assumption of negligible detector noise is satisfied. As described in Saha et al. (2008), this bias could be quantified as $C_{l}^{b(f)}=1 /\left(\mathbf{e}_{0}^{T}\left(\mathbf{C}_{l}^{f}\right)^{-1} \mathbf{e}_{0}\right)$, where $\mathbf{C}_{l}^{f}$ is the foreground covariance matrix. The positive bias increases with the increase in amplitude of foreground components and may be present even at the lowest multipoles when $n_{f} \geqslant n$ is satisfied.

The bias analysis becomes more complex when data are strongly contaminated by the detector noise. In this case the foregrounds remain partly "invisible" due to the presence of detector noise leading to an imperfect foreground removal. A straightforward way to quantify such bias is to compute $\hat{\mathbf{W}}_{l} \mathbf{C}_{l}^{f} \hat{\mathbf{W}}_{l}^{T}$, where $\hat{\mathbf{W}}_{l}$ is the weight vector. A detailed analysis to extract this bias was performed in Saha et al. (2008), assuming the most general covariance structure of the foreground model. The bias is given by

$$
\begin{aligned}
\left\langle\frac{\mathbf{e}_{0}^{T}\left(\hat{\mathbf{C}}_{l}^{N}\right)^{-1}}{\mathbf{e}_{0}^{T}\left(\hat{\mathbf{C}}_{l}^{N}\right)^{-1} \mathbf{e}_{0}} \mathbf{C}_{l}^{f} \frac{\left(\hat{\mathbf{C}}_{l}^{N}\right)^{-1} \mathbf{e}_{0}}{\mathbf{e}_{0}^{T}\left(\hat{\mathbf{C}}_{l}^{N}\right)^{-1} \mathbf{e}_{0}}\right\rangle= & \mathbf{N}_{l} \mathbf{C}_{l}^{f} \mathbf{N}_{l}^{T} \frac{2 l}{2 l+1} \\
& +\frac{\operatorname{tr}\left(\mathbf{C}_{l}^{f}\left(\mathbf{C}_{l}^{N}\right)^{-1}\right)}{(2 l+1)\left(\mathbf{e}_{0}^{T}\left(\mathbf{C}_{l}^{N}\right)^{-1} \mathbf{e}_{0}\right)}
\end{aligned}
$$

where $\hat{\mathbf{C}}_{l}^{N}$ is the empirical detector noise covariance matrix, $\mathbf{C}_{l}^{N}$ is the mean noise covariance matrix, $\mathbf{N}_{l}=$ $\mathbf{e}_{0}^{T}\left(\mathbf{C}_{l}^{N}\right)^{-1} / \mathbf{e}_{0}^{T}\left(\mathbf{C}_{l}^{N}\right)^{-1} \mathbf{e}_{0}$, and $\operatorname{tr}$ denotes trace. Since the second term is weighted down by a factor of $2 l$ compared to the first term, at large $l$ the dominant contribution to the residual comes from the first term. In Saha et al. (2008), Equation (9) was used for estimation of the bias due to the unresolved point sources.

Let us now understand how sensitive the bias correction is to the fidelity of foreground models that may be used to measure the bias using Monte Carlo simulations. We consider the case when detector noise is stronger than the foreground. If the covariance matrix of the foreground components, $\mathbf{C}_{l}^{f}$, used in the simulations is erroneous by a small amount, $\Delta \mathbf{C}_{l}^{f}$, from the true covariance matrix, $\mathbf{C}_{l}^{f 0}$, of the foreground components on the sky, i.e., $\mathbf{C}_{l}^{f 0}=\mathbf{C}_{l}^{f}-\Delta \mathbf{C}_{l}^{f}$, the error in the measured bias can be estimated using Equation (9). The result is given by

$$
\begin{aligned}
& \left\langle\frac{\mathbf{e}_{0}^{T}\left(\hat{\mathbf{C}}_{l}^{N}\right)^{-1}}{\mathbf{e}_{0}^{T}\left(\hat{\mathbf{C}}_{l}^{N}\right)^{-1} \mathbf{e}_{0}}\left(\Delta \mathbf{C}_{l}^{f}\right) \frac{\left(\hat{\mathbf{C}}_{l}^{N}\right)^{-1} \mathbf{e}_{0}}{\mathbf{e}_{0}^{T}\left(\hat{\mathbf{C}}_{l}^{N}\right)^{-1} \mathbf{e}_{0}}\right\rangle \\
& \quad=\mathbf{N}_{l}\left(\Delta \mathbf{C}_{l}^{f}\right) \mathbf{N}_{l}^{T} \frac{2 l}{2 l+1}+\frac{\operatorname{tr}\left(\left(\Delta \mathbf{C}_{l}^{f}\right)\left(\mathbf{C}_{l}^{N}\right)^{-1}\right)}{(2 l+1)\left(\mathbf{e}_{0}^{T}\left(\mathbf{C}_{l}^{N}\right)^{-1} \mathbf{e}_{0}\right)} .
\end{aligned}
$$

As stated earlier, in the large $l$ region the second term contributes negligibly compared to the first term. Assuming that the noise is uncorrelated among different detectors, we find that $\left(\mathbf{C}_{l}^{N}\right)^{-1}$ is a diagonal matrix with positive entries. This implies that each of the components of vector $\mathbf{N}_{l}$ is less than unity. Hence all components of the error covariance matrix, $\Delta \mathbf{C}_{l}^{f}$, of the foreground model, are weighted down before they are added together to give the final error in the bias correction. One can also trivially identify $\mathbf{N}_{l}$ as a vector containing inverse noise weights of different maps. In this case, the least noisy map has the maximum weight. Therefore, error in the bias correction arises dominantly due to error in the foreground model of the least noisy map.

Equation (10) quantifies the uncertainty in the bias correction due to an uncertainty in the foreground model. Since the bias as given by Equation (9) depends linearly upon the foreground covariance matrix, the uncertainty in the bias correction is proportional to the uncertainty in the foreground covariance matrix. Also, the bias correction as well as its uncertainty does not depend upon the CMB amplitude. Using the first and second terms of Equation (10) we conclude that if the detector noise amplitude of certain frequency band is increased the IPSE method assigns a small weight to it, thereby reducing the contribution of this frequency band to the uncertainty in the bias correction.

Now we consider uncertainty in bias that appears when detector noise is negligible and number of foreground components become equal or more than number of available frequency bands. We note that since we always use more frequency bands than the total number of foreground components in Monte Carlo simulations, this situation does not arise in our work. A little algebra gives us the following result for the error in the bias correction due to uncertainty in the foreground model,

$$
\Delta C_{l}^{b(f)}=\mathbf{F}_{l} \Delta \mathbf{C}_{l}^{f} \mathbf{F}_{l}^{T},
$$

where $\mathbf{F}_{l}=\mathbf{e}_{0}^{T}\left(\mathbf{C}_{l}^{f 0}\right)^{-1} / \mathbf{e}_{0}^{T}\left(\mathbf{C}_{l}^{f 0}\right)^{-1} \mathbf{e}_{0}$ and we have performed a matrix expansion up to first order in $\Delta \mathbf{C}_{l}^{f}\left(\mathbf{C}_{l}^{f 0}\right)^{-1}$ assuming $\Delta \mathbf{C}_{l}^{f}\left(\mathbf{C}_{l}^{f 0}\right)^{-1} \ll \mathbf{I}$. Here $\mathbf{I}$ denotes the identity matrix.

As we shall see below, our Monte Carlo simulations suggest that the bias in the IPSE method is generally small. Only for $E E$ power spectrum at low $l$ do we find a somewhat large bias. Therefore, assuming that the true foreground components on the sky are not dramatically different from what we assumed in our simulations, a small change in the magnitude of the foreground model would not change the bias by a very large amount. This is because the bias depends linearly on the foreground covariance matrix. In our simulations we have explicitly verified that the weights corresponding to some chosen combinations of maps for the $E E$ polarization data are very similar to those obtained from the same combination of simulated data. This suggests that the foreground model assumed in simulations cannot be very different from the true foregrounds. We therefore deduce that the error in the bias correction is likely to be small. Furthermore, we explicitly compute the error in the bias estimate for $E E$ power spectrum in Section 3.3.

\section{RESULTS}

\subsection{Temperature Power Spectrum}

The temperature power spectrum for the 5 year WMAP data is obtained using the same procedure as described in Saha et al. (2008). The entire sky is divided into nine regions depending on the level of foreground contamination. The whole cleaning is 


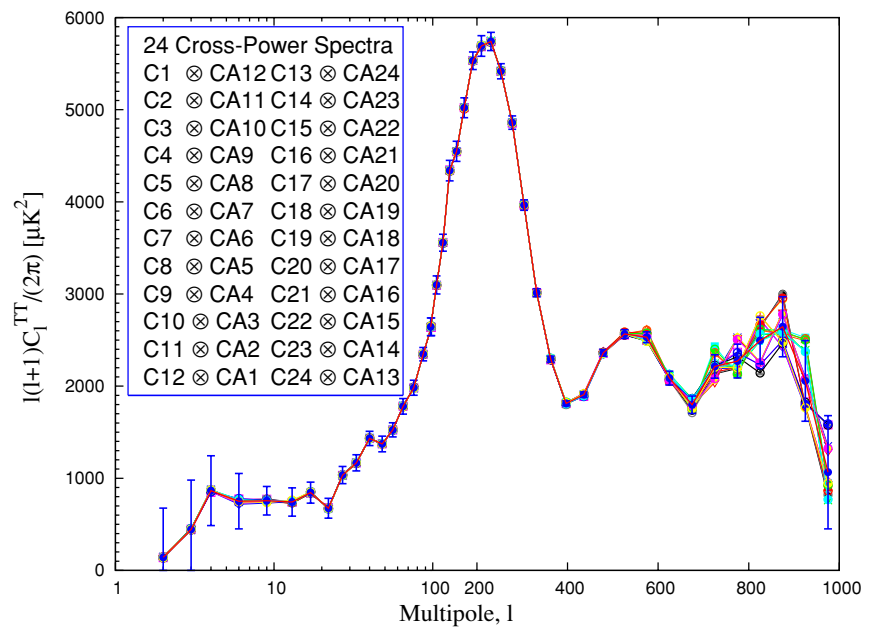

Figure 1. 24 binned, $T T$ cross power spectra obtained by using the WMAP 5 year data. All the different combinations of cleaned maps used are shown in the box. The average power spectrum along with error bars (blue points) is also shown. The red line joins the individual binned averages.

(A color version of this figure is available in the online journal.)

done with the iterative method, starting from the dirtiest region. All the 24 cross power spectra for temperature anisotropy are shown in Figure 1. We form a uniform average power spectrum by averaging over these 24 cross power spectra. While obtaining the cleaned power spectrum we use the KQ85 mask (Gold et al. 2009) to remove the residual foreground contamination near the Galactic plane.

Even after applying the KQ85 mask, which also removes a circular region around each of the known point sources, residual unresolved point sources cause a significant contamination in this power spectrum. There have been several attempts to measure unresolved point source contamination in the CMB maps (Tegmark \& de Oliveira-Costa 1998; Komatsu et al. 2003; Huffenberger et al. 2006). We estimate unresolved point source contamination in our final power spectrum using the model presented by Nolta et al. (2009) following an approach similar to that in Saha et al. (2006, 2008).

We compute the bias in the extracted spectrum by performing 150 Monte Carlo simulations. First we generate synchrotron, free-free, and thermal dust maps corresponding to different WMAP frequencies using the Planck Sky $\mathrm{Model}^{7}$ (PSM). Although several options are available in the PSM to generate galactic emission (e.g., with or without spinning dust, with or without small scales added), the largest scales in temperature are strongly constrained by observation, and the impact of the choice of a particular model is not a major source of uncertainty. In our simulations, we use a single set of galactic emission maps, which comprise a two-component dust model based on the SFD model 7 (Schlegel et al. 1998; Finkbeiner et al. 1999), synchrotron map with varying spectral index in agreement with the first year WMAP data, and free-free emission with fixed spectral index, obtained from an $\mathrm{H} \alpha$ template corrected for galactic dust extinction. The exact polarization properties of the galactic foregrounds, in particular that of dust emission, are poorly constrained by observations. For the present work, we use version 1.6.4 of the PSM (see Betoule et al. 2009 for details about the polarized galactic emission). In the next

\footnotetext{
7 A development version of the PSM can be obtained upon request from the Planck Working Group 2; see http://www.apc.univ-paris7.fr/APC_CS/ Recherche/Adamis/PSM/psky-en.php.
}

step we randomly generate CMB maps assuming the standard LCDM model (Spergel et al. 2003). Each random realization of the $\mathrm{CMB}$ map is then added to the combined mixture of all three foreground components corresponding to the five $W M A P$ frequencies. Using the 5 year beam transform functions for different DAs provided by the WMAP science team, we transform the five resulting maps into ten maps. Each map at this step has a resolution appropriate for the corresponding DA. We then generate random noise maps corresponding to each detector. The random noise maps are generated by sampling a Gaussian distribution with unit variance and then multiplying each Gaussian variable by $\sigma_{0} / \sqrt{N_{p}}$, where $\sigma_{0}$ is the noise per observation (Hinshaw et al. 2009) and $N_{p}$ is the effective number of observations at each pixel. The values of $\sigma_{0}$ depend on the DA, with the smallest value for the $K$-band DA and largest for the $W$-band DAs. Finally, the noise maps are added to the CMB plus foreground maps for different DAs. These maps with CMB signal, detector noise, and foreground are then passed through the same power spectrum estimation method as in the case of observed data. The mean of the 150 extracted spectra gives the final simulated power spectrum. The standard deviation of the 150 simulations gives the error. The difference between the simulated power and the input $L C D M$ power gives a measure of the bias in our method. This bias is subtracted from the extracted power spectrum in order to get the final result.

The precise magnitude of the bias depends on the theoretical model with which we compare our extracted power spectrum. In other words, before we compare our extracted power to a theoretical model, we must correct for bias using the corresponding model power spectrum. Here we use the WMAP best-fit $L C D M$ model to compute the theoretical power spectrum.

The statistical error on the estimate of bias depends on the number of simulations. For 150 simulations the statistical error on bias is equal to $1 / \sqrt{150}$ times the error in the extracted power spectrum. This will lead to a small increase in the estimate of the statistical error on the bias-corrected power spectrum. The statistical error on bias could be reduced by making more simulations but even 150 simulations appear sufficient for a reliable estimate. The bias estimate may also have significant systematic error. This is important for the case of polarization power spectrum, where, as we shall see, the bias is relatively large for some of the bins. We make an estimate of the systematic error in the bias for the $E E$ power spectrum in Section 3.3.

The final temperature power spectrum using the IPSE method, after correcting for bias, is shown in Figure 2. We find that it is in good agreement with the WMAP best-fit LCDM model. The simulation results are also shown in Figure 2. After bias correction we find the quadrupole power $\left(l(l+1) C_{l} / 2 \pi\right)$ equal to $532 \mu \mathrm{K}^{2}$ compared to the value of $213.4 \mu \mathrm{K}^{2}$ estimated by the WMAP science team. Our result, $532 \mu \mathrm{K}^{2}$, is different from what is given in Saha et al. (2006) since the bias correction was not applied in Saha et al. (2006). The quadrupole extracted from IPSE is, therefore, in much better agreement with the theoretical model.

We point out that our theoretical bias estimate at low $l$, obtained using the formula $-c C_{l}^{c} /(2 l+1)$, given in Section 2.1, is higher in comparison to the result obtained by direct numerical simulations. The difference arises since the weights are estimated using a smoothed version of the covariance matrix, $\hat{\mathbf{C}}_{l}$, during the foreground removal stage. In particular, we perform a running average of the covariance matrix using a window function of width $\delta_{l}=11$. Such smoothing reduces the random chance correlation power of CMB and foreground components. 

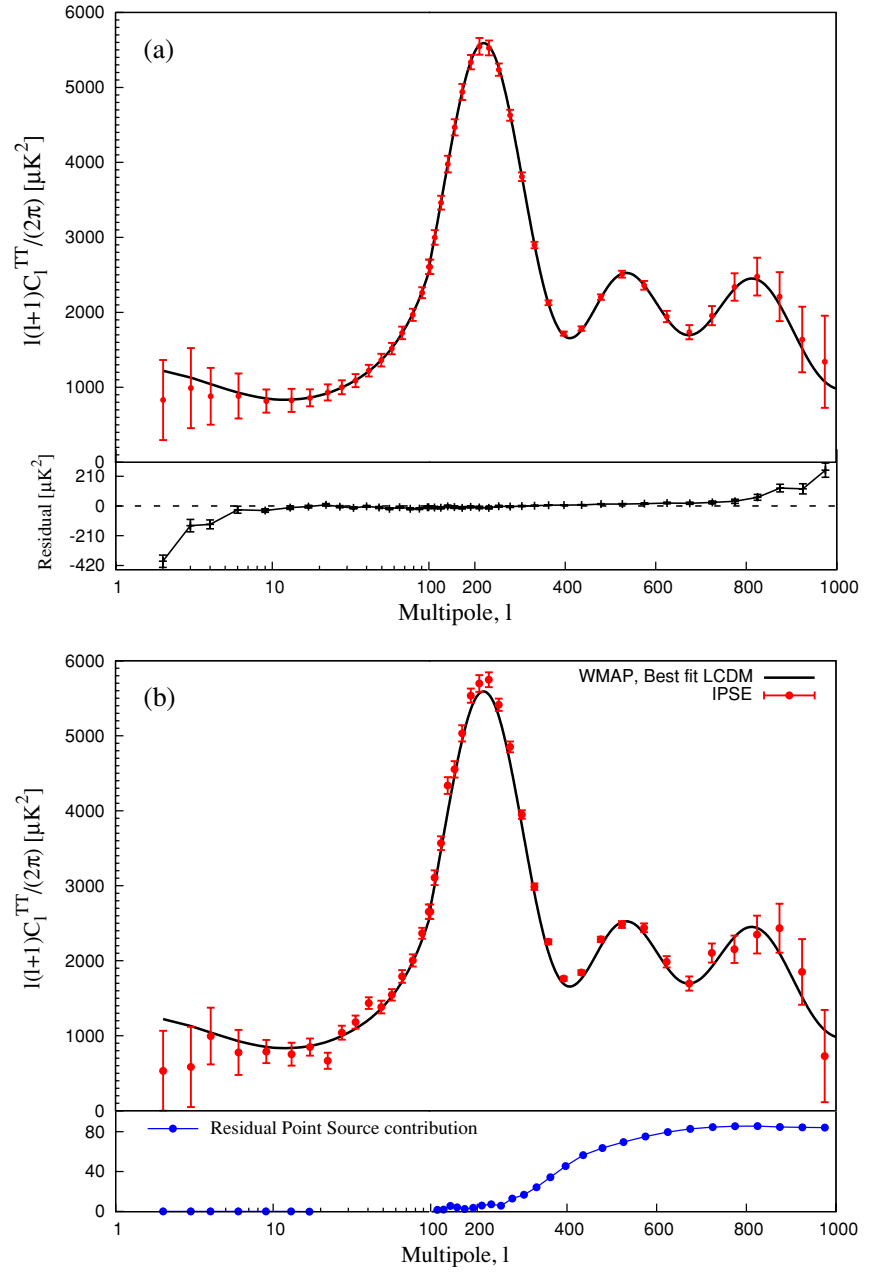

Figure 2. (a) Simulation result for the $T T$ power spectrum (red dots), along with error bars, using IPSE. The WMAP LCDM best-fit model (black line) is shown for comparison. The bottom panel shows the residuals along with the simulation standard errors. (b) The final, binned, $T T$ power spectrum using IPSE (red dots) along with error bars for the 5 year WMAP data, after subtracting the bias extracted using simulations. The error bars are also obtained from simulations. The WMAP LCDM best-fit model (black line) is shown for comparison. The bottom panel shows the correction made for residual power from unresolved point source contamination

(A color version of this figure is available in the online journal.)

Hence the negative bias at low $l$ obtained from simulation turns out to be smaller in comparison to the analytical estimate, which is derived without any assumption of smoothing on the covariance matrix.

The correlation matrix, Equation (8), for temperature power spectrum is shown in Figure 3. We find, as expected, that the off-diagonal matrix elements are negligible compared to the diagonal elements.

\subsection{The TE Power Spectrum}

The WMAP polarization CMB maps are cleaned using a single iteration rather than the nine-iteration procedure followed for temperature anisotropy. We apply the P06 mask right in the beginning. The mask removes $27 \%$ of the entire sky region near the galactic plane. The cleaning algorithm is applied only to regions outside the P06 mask. Hence we make no attempt to produce a full-sky cleaned polarization map.

The error bars plotted in the $T E$ power spectrum are obtained by Monte Carlo simulations. We generate 150 random samples

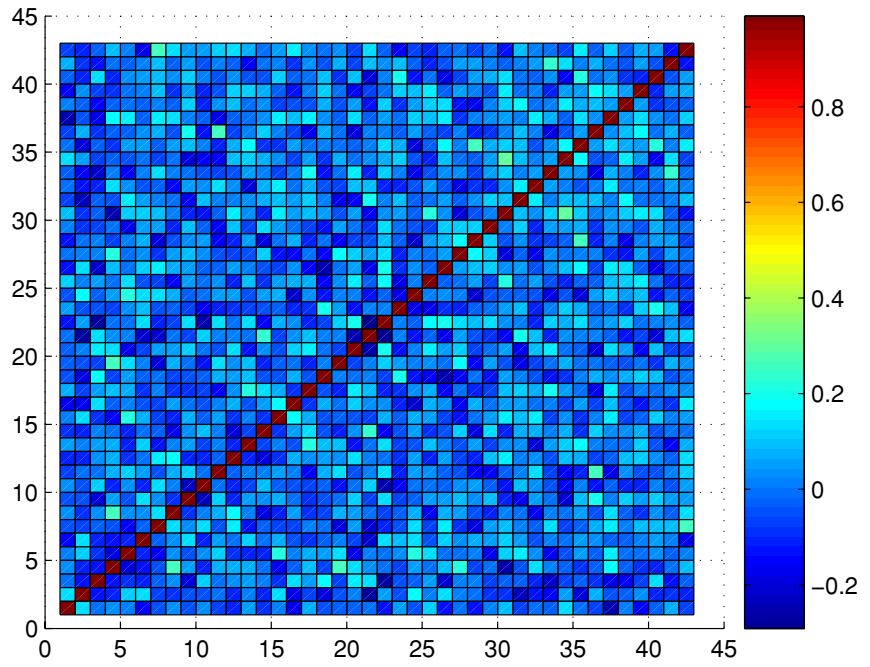

Figure 3. Correlation matrix elements, $C_{\alpha \alpha^{\prime}}$, defined in Equation (8), for the temperature power spectrum, plotted with respect to the bin indices $\alpha, \alpha^{\prime}$.

(A color version of this figure is available in the online journal.)

of data using the LCDM model (Spergel et al. 2003) and simulated foregrounds and detector noise. First we generate the synchrotron and thermal dust polarized foreground maps corresponding to different frequencies in terms of $Q$ and $U$ maps using the PSM version 1.6.4. The free-free emission is not polarized and hence not included. The anomalous dust emission is also assumed to be unpolarized and thus is not included either. Using the HEALPix ${ }^{8}$ command synfast we generate random realization of CMB polarization maps in terms of $Q$ and $U$ maps. The random $\mathrm{CMB}$ realization and foreground maps are smoothed by the beam functions corresponding to ten different DAs. We next obtain $E$-mode polarization maps from these $Q$ and $U$ maps. Then we generate random noise maps for each DA in terms of Stokes parameters $Q$ and $U$ using Cholesky decomposition technique for generating correlated Gaussian random variables using the WMAP supplied $2 \times 2 Q U$ intrapixel noise covariance matrices. These $Q$ and $U$ noise maps are converted to $E$-mode noise maps. The final $E$-maps including detector noise, foregrounds, and CMB signal are passed through the same cleaning pipeline as the observed polarization data. In order to minimize the correlation among neighboring $l$ modes, the final power spectrum is binned in the same way as the WMAP 5 year result. Here also the standard deviation obtained from the diagonal elements of the binned covariance matrix is used as the error bars on the binned final spectrum extracted from the WMAP data.

The extracted $T E$ power spectrum along with the WMAP results and the best-fit LCDM model is shown in Figure 4. The binned $T E$ power spectrum, using the same binning scheme as used by the WMAP team, is shown in Figure 5. The error bars are computed by simulations. The simulation results are shown in Figure 6. The bottom panel of this figure shows the residuals, i.e., the difference of the simulation and the model $L C D M$ power. The error bars on the residuals correspond to the simulation standard error. For 150 simulations we find that the simulation error is sufficiently small so that it can be neglected. We find that the bias is small for all the bins. Only at small $l, l<10$, do we find a noticeable negative bias. For larger $l$, the bias is practically negligible. The

\footnotetext{
8 http://healpix.jpl.nasa.gov
} 


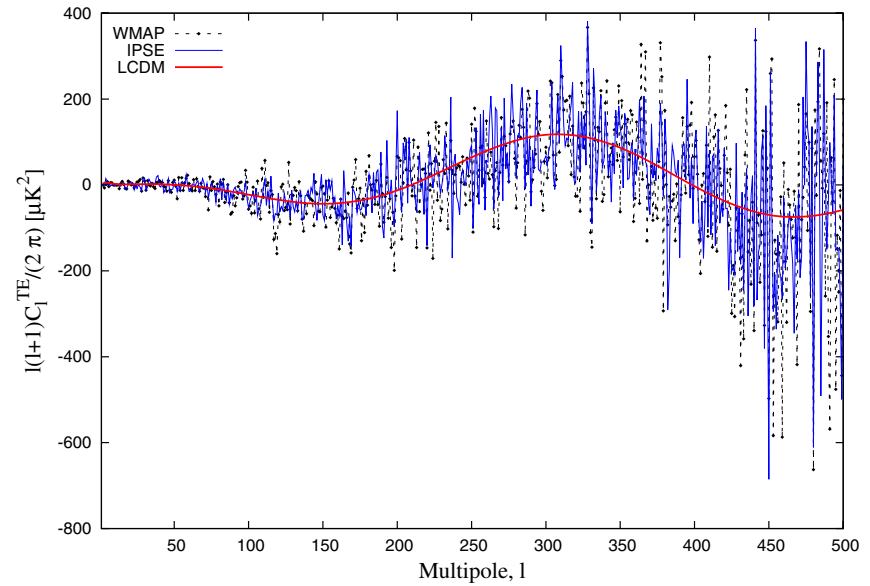

Figure 4. Cleaned $T E$ power spectrum using IPSE (solid blue line) along with $W M A P$ result (dashed black line). The $W M A P$ best-fit $L C D M$ power spectrum (thick solid red line) is shown for comparison.

(A color version of this figure is available in the online journal.)

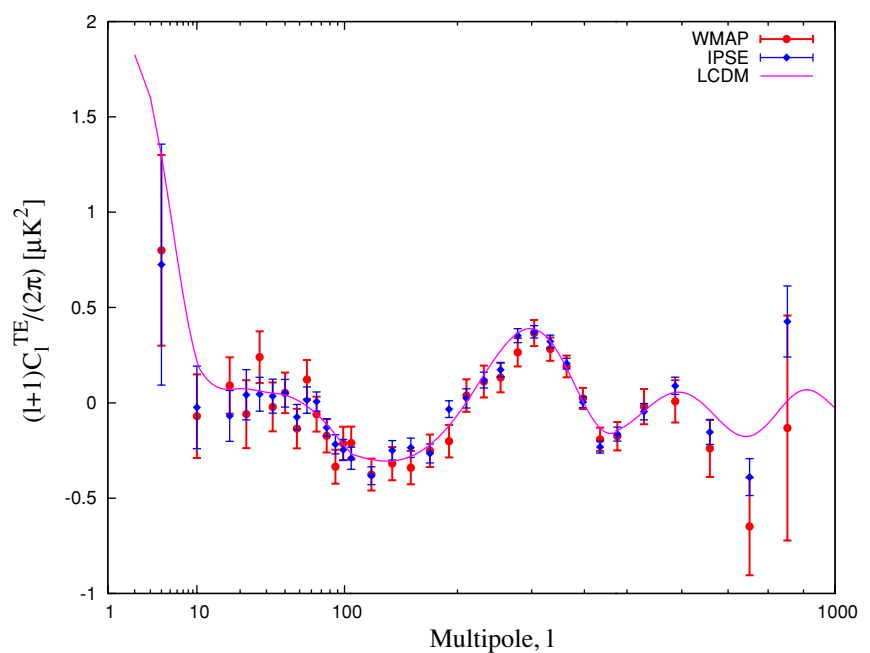

Figure 5. Final binned $T E$ power spectrum using IPSE (blue diamonds) along with error bars, compared with the WMAP results (red dots). The WMAP best-fit $L C D M$ result (solid pink line) is also shown.

(A color version of this figure is available in the online journal.)

bias-corrected $T E$ power spectrum is shown in Figure 7. The spectrum obtained by the WMAP science team as well as their best-fit $L C D M$ model is also shown. We find good agreement with the WMAP result. However, we obtain slightly smaller error bars. We discuss the possible cause for this in the next subsection. The correlation matrix elements, Equation (8), are shown in Figure 8. Here also we see that they are dominated by diagonal elements.

\subsection{The EE Power Spectrum}

The binned $E E$ power spectrum, using the WMAP binning procedure, along with the simulation results, is shown in Figure 9 for the 5 year data. In this figure, we also show the $E E$ power spectrum extracted by the WMAP team along with the WMAP best-fit $L C D M$ model. The lower panel of this figure shows the simulation residuals, i.e., the difference between the simulation results and the $L C D M$ power, along with the corresponding standard error. In Figure 9, we follow exactly the WMAP binning procedure and consider only the multipoles $l \geqslant 50$. The results for low multipoles $l<50$ are shown separately. We

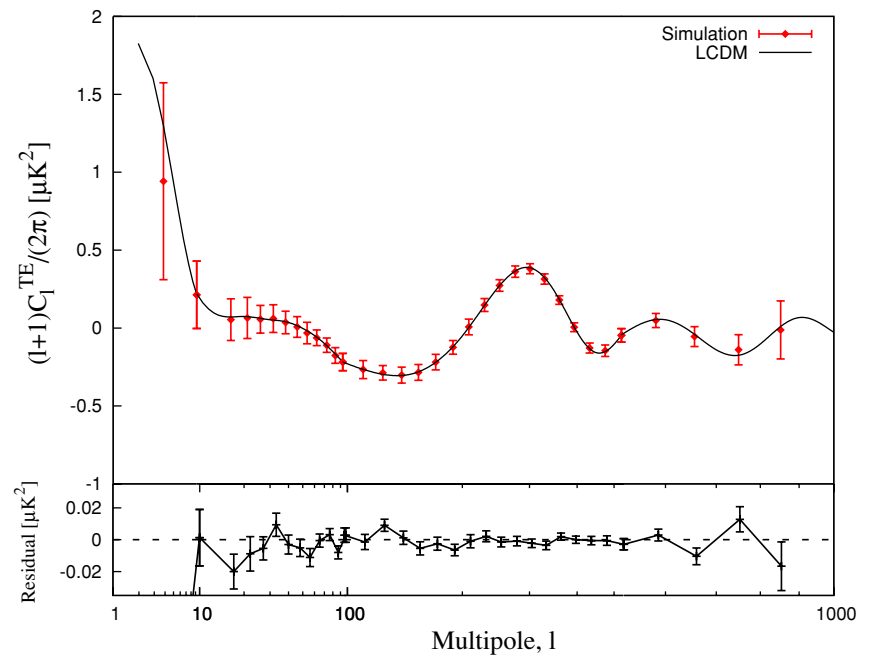

Figure 6. Binned $T E$ power spectrum, along with error bars, obtained from simulations (red dots). The input LCDM model (solid black line) is also shown. The bottom panel shows the residuals along with the simulation standard error. (A color version of this figure is available in the online journal.)

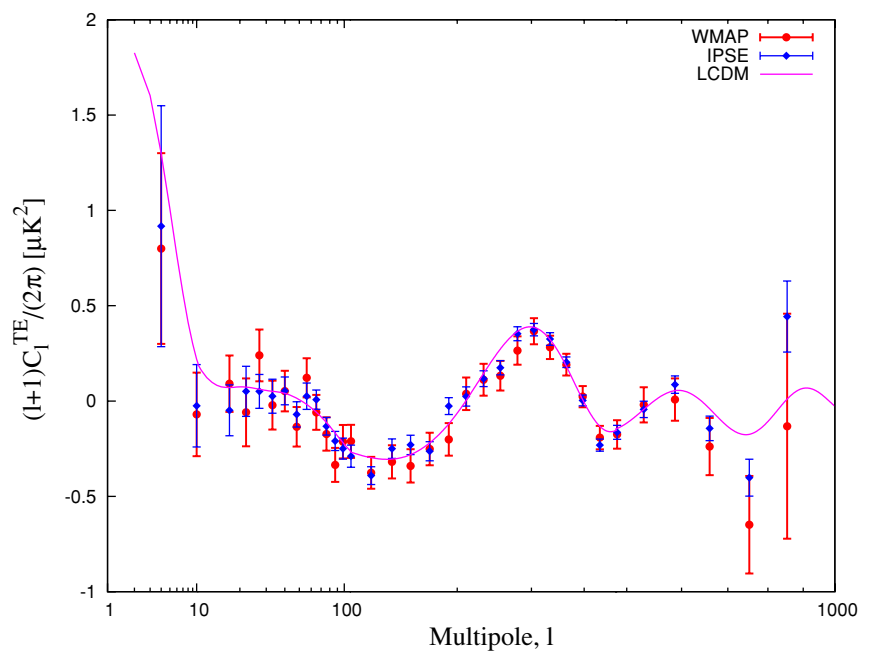

Figure 7. Bias-corrected, binned $T E$ power spectrum using IPSE (blue diamonds) along with error bars, compared with the WMAP results (red dots). The $W M A P$ best-fit $L C D M$ power spectrum (solid pink line) is also shown.

(A color version of this figure is available in the online journal.)

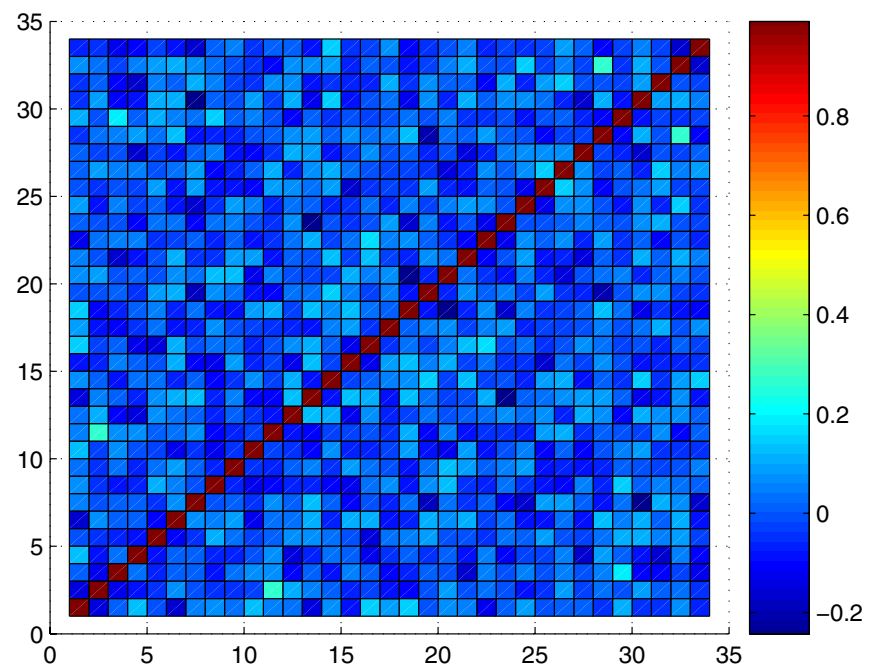

Figure 8. Covariance matrix elements, $C_{\alpha \alpha^{\prime}}$, defined in Equation (8), for the $T E$ power spectrum plotted with respect to the bin indices $\alpha, \alpha^{\prime}$.

(A color version of this figure is available in the online journal.) 


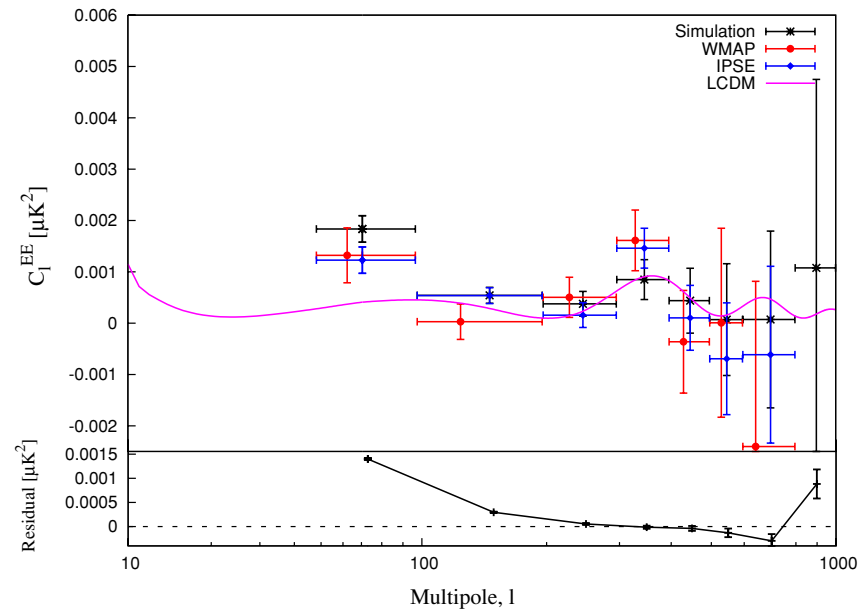

Figure 9. CMB polarization $E E$ power spectrum using IPSE (blue diamonds) along with error bars, compared with the WMAP result (red dots). The WMAP best-fit $L C D M$ power spectrum (pink solid line) and the simulation results (black crosses) are also shown. The bottom panel shows the simulation residuals along with the corresponding standard errors.

(A color version of this figure is available in the online journal.)

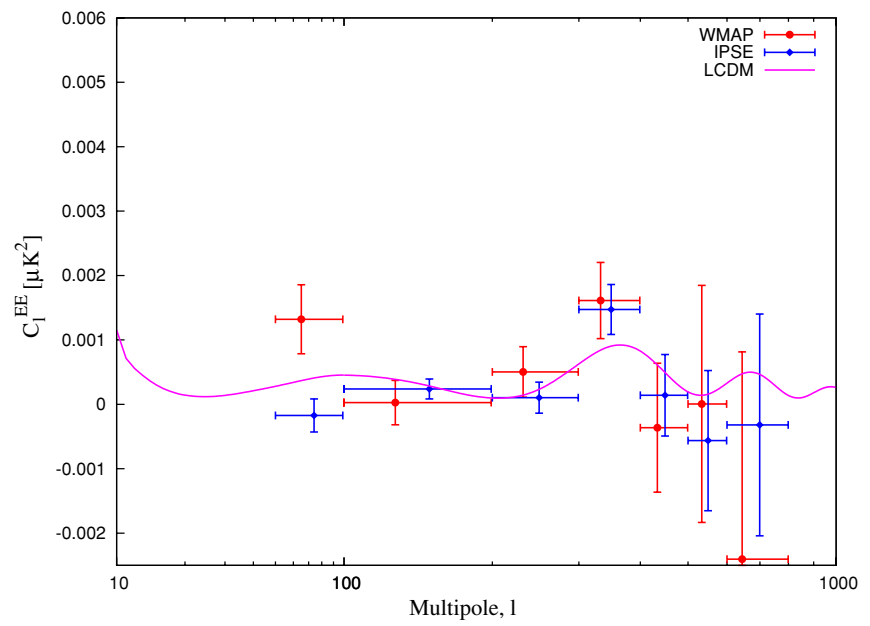

Figure 10. CMB polarization $E E$ power spectrum using IPSE (blue diamonds) along with error bars, after correcting for bias. The WMAP result (red dots) and the theoretical $L C D M$ spectrum (pink solid line) are shown for comparison.

(A color version of this figure is available in the online journal.)

find that our extracted power spectrum is in good agreement with that obtained by the WMAP team but with somewhat smaller error bars. Furthermore, the binned simulated power spectrum is found to be close to the input $L C D M$ power over the entire multipole range. Only at small $l$ do we find a significant positive bias. For the remaining multipoles the simulation results match the input power within error bars. The bias-corrected power spectrum is shown in Figure 10. We see that the biascorrected spectrum is in reasonable agreement with the best-fit LCDM model. The correlation matrix elements are shown in Figure 11. We again find that the correlation matrix is dominated by diagonal matrix elements.

Our simulation results for low $l(l \leqslant 50)$ are shown in Figure 12. The WMAP power spectrum as well as their best-fit LCDM model is also shown. Here we have chosen the binning that was used by the WMAP team for the TE power spectrum. The bias-corrected power spectrum is shown in Figure 13. We find that at very low $l \leqslant 10$, we have significantly higher scatter in comparison to the results obtained by WMAP. Here our error bars are also slightly larger in comparison to WMAP. The cause

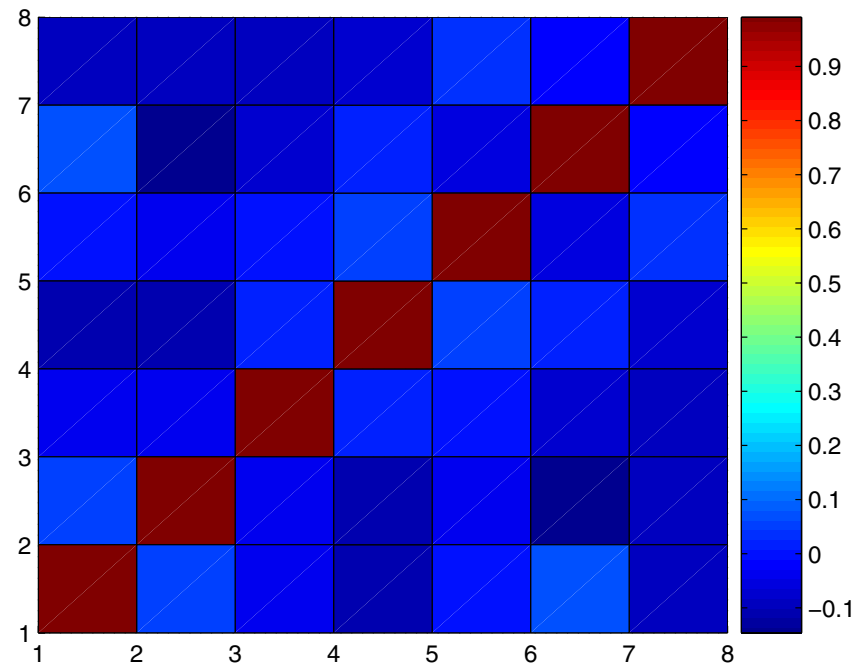

Figure 11. Correlation matrix elements, $C_{\alpha, \alpha^{\prime}}$, defined in Equation (8), for the $E E$ power spectrum plotted with respect to the bin indices $\alpha, \alpha^{\prime}$.

(A color version of this figure is available in the online journal.)

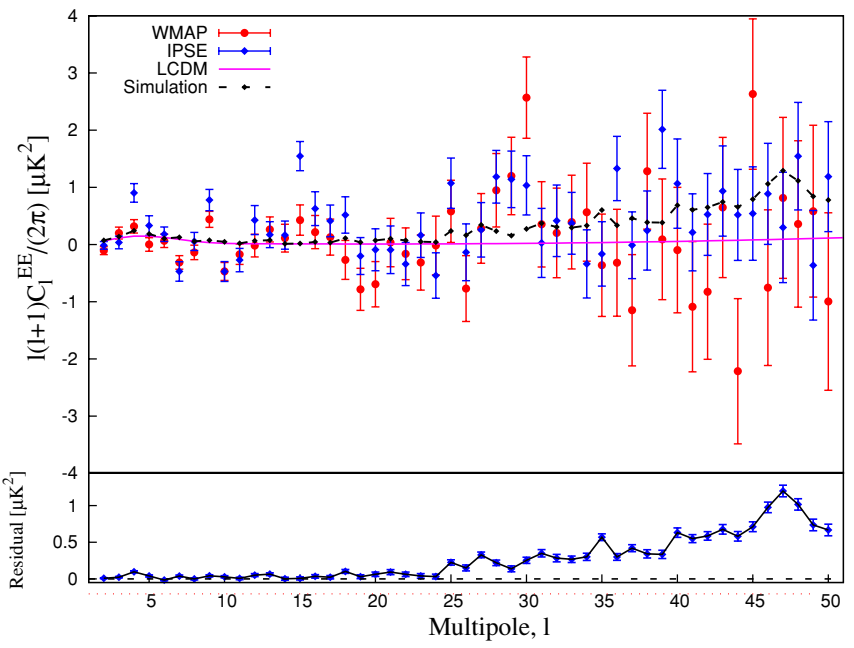

Figure 12. Binned $E E$ power spectrum using IPSE (blue diamonds) at low- $l$ along with the results by the WMAP science team (red dots). The theoretical $L C D M$ spectrum (solid pink line) and the ensemble averaged $E E$ power spectrum from simulated data (dashed black line) are also shown. The bottom panel shows the simulation residuals along with the standard error.

(A color version of this figure is available in the online journal.)

of this scatter is so far unknown. At larger $l$ we find smaller scatter and error bars in comparison to WMAP.

The extracted bias in the case of $E E$ power spectrum is relatively large especially at low $l$. Hence it is useful to estimate the uncertainty in the calculation of bias due to the uncertainty in foregrounds and the detector noise. The polarized foregrounds are not known very well. The error in the individual foreground components may be quite large. However the error in the total foreground power at low $l$, where the foregrounds dominate, is expected to be about $1 \%$. The estimate $1 \%$ is obtained by comparing the simulated power, using PSM foreground model, with the power observed by WMAP. The difference in power spectrum, outside the P06 mask, for the lowest $l$ bin, $l=74$, is found to be of the order of $1 \%$. At the binned multipole $l=149$ we also find a similar error. The difference at all other bins is found to be smaller.

In order to determine the error due to foregrounds we assume a $1 \%$ error in the synchrotron contribution to the foregrounds. We point out that this is the dominant component of the foregrounds, 


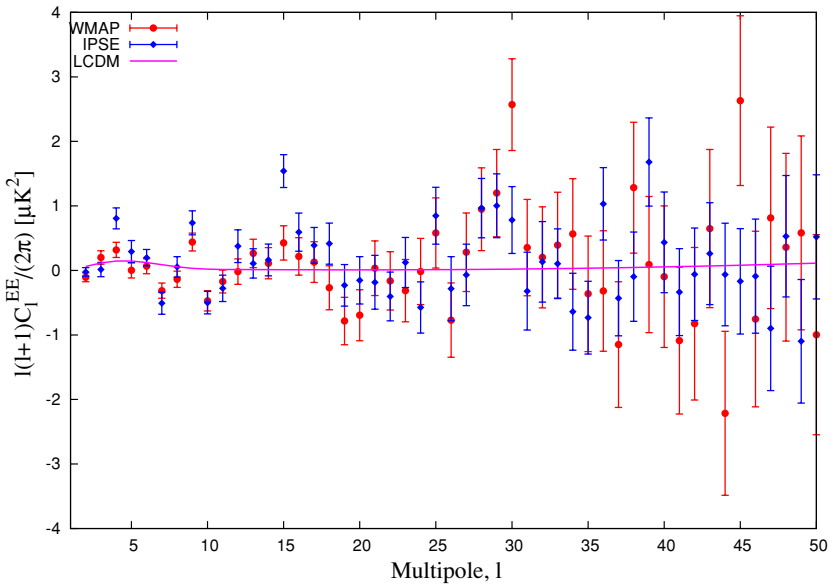

Figure 13. Bias-corrected $E E$ power spectrum using IPSE (blue diamonds) at low $l$ compared with the WMAP results (red dots). The WMAP best-fit LCDM power spectrum (pink line) is also shown.

(A color version of this figure is available in the online journal.)

expected to contribute of the order of $99 \%$ of the foregrounds. By direct simulations, the error in the bias is found to be $0.88 \%$, $1.02 \%, 0.30 \%, 0.03 \%, 0.02 \%, 0.04 \%, 0.02 \%$, and $0.0006 \%$ for the bin centered at 74, 149, 249, 349, 449, 549, 699, and 899 , respectively. At very low $l(l<15)$ we find that the corresponding error in the bias is less than $0.1 \%$. Hence we find that a $1 \%$ increase of synchrotron foreground level leads to a maximum of $1 \%$ error in the bias. Since the polarized dust foreground level is significantly smaller than the level of synchrotron emission outside P06 mask we find that the error in the estimated bias due to the $1 \%$ error in dust foreground level is less than $0.03 \%$. Hence we conclude that a $1 \%$ error in the foreground power leads to a maximum of $1 \%$ error in the estimated bias. We point out that a $1 \%$ uncertainty in the foregrounds amounts to a very large uncertainty in the power in comparison to the $\mathrm{CMB}$ signal. In contrast, a $1 \%$ uncertainty in the bias is very small. Hence, the uncertainty in our final power spectrum is a very small fraction of the uncertainty in the foreground power used for simulations.

We next estimate the error in the bias due to uncertainty in the noise level. We perform Monte Carlo simulations by increasing the noise level per observation, $\sigma_{0}$, for different $W M A P$ bands by $0.1 \%$, consistent with the WMAP team's estimated uncertainty in these values. In this case we find a significantly smaller error in the bias estimation. The maximum error was found to be $0.1 \%$ for the multipole bin centered at $l=699$. We, therefore, find that the maximum error in the estimation of the bias due to the error in foregrounds and detector noise is of the order of $1 \%$. Hence our method is expected to be sufficiently reliable for cosmological parameter estimation.

As discussed in Section 3.1 the precise magnitude of bias depends on the theoretical model with which we compare our extracted power spectrum. Here we determine the change in bias due to a $1 \%$ change in the cosmological model. We find that for low $l$ bins $(l<150)$ the error in the bias is less than $1 \%$. The error starts increasing with $l$ reaching a maximum of $6 \%$ for the bin centered at $l=699$. Beyond this the error again starts to decrease rapidly. Hence in this case we find a relatively large error of the order of $6 \%$ for some of the bins. However, at these bins the overall bias is very small, as can be seen from Figure 9 . Hence a $6 \%$ error in the bias leads to a relatively small error in the overall power. Furthermore, it should be possible to iteratively improve the estimates of the model parameters which provide the best fit to the extracted power spectrum, after correcting for bias. We start by obtaining the cosmological model parameters using the extracted power spectrum, which is so far uncorrected for bias. Next we obtain the bias correction using the model parameters. After correcting for bias we obtain the power spectrum which can be used to obtain an improved estimate of model parameters. Hence, the uncertainty in the bias correction due to uncertainty in the model parameters may be considerably reduced by this iterative procedure.

These Monte Carlo simulations verify that the error in the bias is directly proportional to the error in the foreground power in the noise dominated regime, but does not depend strongly on the error in the detector noise model. This result may also be obtained by considering the first term in Equation (10), as discussed in the text following this equation. We find that the maximum error in bias due to the error in foregrounds is found in the intermediate range where the foreground power is comparable to noise. At low $l$ the foreground power is large in comparison to the noise whereas the noise dominates at large $l$. In both these regimes the error in the bias is very small. We expect that the IPSE method would perform very well at low $l$ for Planck HFI frequency maps since in this case the detector noise is expected to be small compared to WMAP.

Since the power spectrum estimation methodology described in this work is fundamentally different from WMAP team's approach we should not expect both methods to produce identical error bars on the derived power spectra, although the power spectra themselves, obtained by using the two methods, are in reasonable agreement with each other. We find that our error bars on the polarization power spectra are generally smaller compared to those obtained by the WMAP science team except at very low $l$. Our error bars on $E E$ power spectrum at $l \leqslant 15$ are somewhat larger in comparison to that obtained by WMAP. The error bars get negligible contribution from the cosmic variance and are dominated by instrumental noise. Our smaller error bars could be explained by noting that we use more detector maps for polarization power spectrum estimation in comparison to the $W M A P$ science team. Using more detectors increases the signalto-noise ratio of the cleaned map by decreasing the effective noise level. The reduced noise level leads to lower error bars on our polarization power spectra. Furthermore, in the case of noisy polarization data the weights tend to be inversely proportional to the noise. Due to this inverse noise weighting each cleaned map has lesser noise in comparison to the least noisy $K$ - or $K a$-band maps. This may be another reason for smaller error bars in the case of polarization power spectrum.

We are so far unable to determine the cause of the larger scatter and corresponding error bars at $l \leqslant 15$. We have explicitly verified that the larger scatter remains even if we exclude the $W$ band from our analysis. Hence we cannot attribute this to a systematic error in the $W$ band. We find that if we use an enhanced mask then the scatter gets reduced but does not get completely eliminated. This suggests that the problem may arise partially due to the presence of some residual foregrounds. Another possible reason for larger scatter at low $l$ is that the number of $a_{l m}$ 's per $l$ is smaller at low $l$ in comparison with high $l$. This implies that the foreground removal procedure may introduce more noise at low $l$ since effectively the ratio of the number of parameters or weights to the number of observations is larger at low $l$. A detailed study of this issue is required, and which we postpone for future research.

Finally, we determine how the polarization results change if we remove the $W$ band from our analysis. As expected, we find 
that the results show significantly enhanced scatter in almost the entire multipole range. This is consistent with our argument that IPSE yields smaller error bars partially due to the inclusion of more number of bands.

\section{CONCLUSION}

We have used a model-independent method to estimate the $\mathrm{CMB}$ temperature and polarization power spectrum using WMAP 5 year data. The method is based on the assumption that the CMB signal is independent of frequency in thermodynamic temperature unit. Since the foregrounds are frequency dependent in this unit, it is possible to minimize the foreground power by making a linear combination of CMB maps with a suitable choice of weights. For foreground minimization we use the CMB maps in harmonic space. For the case of polarization, the raw full-sky $Q$ and $U$ maps are first converted to $E$ and $B$ maps to avoid any mixing of $E$ and $B$ modes. The total number of maps available for each field for WMAP data is ten, corresponding to the ten DAs. We create several cleaned maps by choosing different subsets of ten DAs, such that each set contains only four DAs. The detector noise power is minimized by crosscorrelating cleaned maps obtained from distinct DAs. This leads to a considerable reduction of the detector noise power since, to a good approximation, noise is uncorrelated among different detectors.

We find that the error bars on our $T E$ and $E E$ power spectra are smaller than those obtained by the WMAP science team on almost all the angular scales. The only exception to this is the $E E$ power spectrum for very low $l$ where we find larger error bars. The low error bars arise mainly because we utilize all ten $W M A P$ DA maps to estimate the polarization power spectra. Another possible reason is that in a noisy data the weights tend to combine the maps in an inverse noise weighted manner. This results in each cleaned map having lesser noise than the least noisy $K$ - or $K a$-band maps.

In the case of the $T T$ power spectrum we find that our procedure does not remove all the unresolved point source contamination. This contamination is significant at small angular scales where the detector noise is also very large. Hence here our internal cleaning is not very efficient. The residual unresolved point source contamination is removed by using the WMAP point source model, as described in detail in Saha et al. (2006, 2008).

We have performed detailed simulations of the $T T, T E, E E$ power spectrum, using the WMAP best-fit $L C D M$ model, along with the foreground and detector noise models, in order to determine if there exists any bias in the extracted power. In all cases the bias is found to be small for the entire multipole range. The extracted power, with or without bias correction, is found to be in good agreement with the WMAP results. In Saha et al. (2008), the authors noticed a negative bias at low $l$ in the $T T$ power spectrum. The negative bias arises due to a chance correlation between the $\mathrm{CMB}$ and the foregrounds. After correcting for the negative bias, we find that the quadrupole for the WMAP 5 year data shows much better agreement with the LCDM model, in comparison to the result obtained by the WMAP science team. Excluding $l=2$, we find negligible bias at all multipoles except at very large $l$ values, where we find a small positive bias. For the case of the $T E$ power spectrum we also find a small negative bias at low $l, l<10$. For larger $l$ values the bias is negligible. For the $E E$ power spectrum also, the bias is found to be small compared to the corresponding error bars. A significant positive bias is found only at low $l$.
To summarize, we have performed a completely independent re-analysis of WMAP 5 year temperature and polarization data. Our procedure uses primarily the CMB data. Hence, it is free from any bias that might result from the inadequacies and inaccuracies of the foreground modeling. The foreground templates and detector noise modeling is utilized only for the purpose of bias analysis and error estimation. The bias is found to be small for all the spectra over the entire multipole range. Our results verify the basic power spectra results obtained by the WMAP Science team. We expect that the method will be very useful for analyzing data from future CMB probes such as Planck.

We acknowledge the use of the Legacy Archive for Microwave Background Data Analysis. Some of the results of this work are derived using the publicly available HEALPIx package (Górski et al. 2005). (The HEALPIX distribution is publicly available from the Web site http://healpix.jpl.nasa.gov.) We acknowledge the use of the PSM, developed by the Component Separation Working Group (WG2) of the Planck Collaboration. Pramoda K. Samal acknowledges CSIR, India for financial support under the research grant CSIR-SRF- 9/92(340)/2004EMR-I. A portion of the research described in this paper was carried out at the Jet Propulsion Laboratory, California Institute of Technology, under a contract with the National Aeronautics and Space Administration.

\section{REFERENCES}

Bardeen, J. M., Steinhardt, P. J., \& Turner, M. S. 1983, Phys. Rev. D, 28, 679 Basko, M. M., \& Polnarev, A. G. 1980, Sov. Astron., 24, 268

Bennett, C. L., et al. 2003a, Astrophys. J. Series, 148, 1

Bennett, C. L., et al. 2003b, ApJS, 148, 97

Betoule, M., Pierpaoli, E., Delabrouille, J., Le Jeune, M., \& Cardoso, J.-F. 2009, A\&A, 503, 691

Bond, J. R., Efstathiou, G., \& Tegmark, M. 1997, MNRAS, 291, L33

Bouchet, F., et al. 1995, New Astron., 4, 443

Bunn, E. F., Zaldarriaga, M., Tegmark, M., \& de Oliveira-Costa, A. 2003, Phys. Rev. D, 67, 023501

Crittenden, R., Davis, R. L., \& Steinhardt, P. J. 1993, ApJ, 417, L13

Crittenden, R. G., Coulson, D., \& Turok, N. G. 1995, Phys. Rev. D, 52, 5402

Delabrouille, J., \& Cardoso, J.-F. 2009, in Lecture Notes in Physics 665, Data Analysis in Cosmology, ed. V. Martinez et al. (Berlin: Springer), 159

Delabrouille, J., Cardoso, J.-F., Le Jeune, M., Betoule, M., Fay, G., \& Guilloux, F. 2009, A\&A, 493, 835

Eriksen, H. K., Banday, A. J., Górski, K. M., \& Lilje, P. B. 2004, ApJ, 612, 633

Eriksen, H. K., Dickinson, C., Jewell, J. B., Banday, A. J., Górski, K. M., \& Lawrence, C. R. 2008a, ApJ, 672, L87

Eriksen, H. K., Huey, G., Banday, A. J., Górski, K. M., Jewell, J. B., O’Dwyer, I. J., \& Wandelt, B. D. 2007a, ApJ, 665, L1

Eriksen, H. K., et al. 2007b, ApJ, 656, 641

Eriksen, H. K., Jewell, J. B., Dickinson, C., Banday, A. J., Górski, K. M., \& Lawrence, C. R. 2008b, ApJ, 676, 10

Finkbeiner, D. P., Davis, M., \& Schlegel, D. J. 1999, ApJ, 524, 867

Fixsen, D. J., Cheng, E. S., Gales, J. M., Mather, J. C., Shafer, R. A., \& Wright, E. L. 1996, ApJ, 473, 576

Fosalba, P., \& Szapudi, I. 2004, ApJ, 617, L95

Ghosh, T., Saha, R., Jain, P., \& Souradeep, T. 2009, Phys. Rev. D, 79, 123011

Gold, B., et al. 2009, ApJS, 180, 265

Górski, K. M., Hivon, E., Banday, A. J., Wandelt, B. D., Hansen, F. K., Reinecke, M., \& Bartelmann, M. 2005, ApJ, 622, 759

Guth, A. H., \& Pi, S.-Y. 1982, Phys. Rev. Lett., 49, 1110

Harari, D. D., \& Zaldarriaga, M. 1993, Phys. Lett. B, 319, 96

Hill, R. S., et al. 2009, ApJS, 180, 246

Hinshaw, G., et al. 2003, ApJS, 148, 135

Hinshaw, G., et al. 2009, ApJS, 180, 225

Hivon, E., Górski, K. M., Netterfield, C. B., Crill, B. P., Prunet, S., \& Hansen, F. 2002, ApJ, 567, 2

Huffenberger, K. M., Eriksen, H. K., \& Hansen, F. K. 2006, ApJ, 651, L81

Jaffe, A. H., Kamionkowski, M., \& Wang, L. 2000, Phys. Rev. D, 61, 083501 Jarosik, N., et al. 2003, ApJS, 148, 29 
Jarosik, N., et al. 2007, ApJS, 170, 263

Jungman, G., Kamionkowski, M., Kosowsky, A., \& Spergel, D. N. 1996a, Phys. Rev. D, 54, 1332

Jungman, G., Kamionkowski, M., Kosowsky, A., \& Spergel, D. N. 1996b, Phys. Rev. Lett., 76, 1007

Kamionkowski, M., \& Kosowsky, A. 1998, Phys. Rev. D, 57, 685

Kamionkowski, M., Kosowsky, A., \& Stebbins, A. 1997, Phys. Rev. D, 55, 7368

Kim, J., Naselsky, P., \& Christensen, P. R. 2008, Phys. Rev. D, 77, 103002

Kim, J., Naselsky, P., \& Christensen, P. R. 2009, Phys. Rev. D, 79, 023003

Kinney, W. H. 1998, Phys. Rev. D, 58, 123506

Kogut, A., et al. 2007, ApJ, 665, 355

Komatsu, E., et al. 2003, Astrophys. J. Sci., 148, 119

Komatsu, E., et al. 2009, ApJS, 180, 330

Lue, A., Wang, L., \& Kamionkowski, M. 1999, Phys. Rev. Lett., 83, 1506

Mather, J. C., et al. 1994, ApJ, 420, 439

Ng, K. L., \& Ng, K.-W. 1995, Phys. Rev. D, 51, 364

Nolta, M. R., et al. 2009, ApJS, 180, 296

Page, L., et al. 2007, Astrophys. J. Sci., 170, 335

Patanchon, G., Cardoso, J.-F., Delabrouille, J., \& Vielva, P. 2005, MNRAS, 364 , 1185
Rees, M. J. 1968, ApJ, 153, L1

Saha, R., Jain, P., \& Souradeep, T. 2006, ApJ, 645, L89

Saha, R., Prunet, S., Jain, P., \& Souradeep, T. 2008, Phys. Rev. D, 78, 023003

Schlegel, D. J., Finkbeiner, D. P., \& Davis, M. 1998, ApJ, 500, 525

Seljak, U., \& Zaldarriaga, M. 1997, Phys. Rev. Lett., 78, 2054

Smith, K. M., \& Zaldarriaga, M. 2007, Phys. Rev. D, 76, 043001

Souradeep, T., Saha, R., \& Jain, P. 2006, New Astron. Rev., 50, 854

Spergel, D. N., \& Zaldarriaga, M. 1997, Phys. Rev. Lett., 79, 2180

Spergel, D. N., et al. 2003, ApJS, 148, 175

Starobinsky, A. A. 1982, Phys. Lett. B, 117, 175

Tegmark, M., \& de Oliveira-Costa, A. 1998, ApJ, 500, L83

Tegmark, M., \& de Oliveira-Costa, A. 2001, Phys. Rev. D, 64, 063001

Tegmark, M., de Oliveira-Costa, A., \& Hamilton, A. J. 2003, Phys. Rev. D, 68 123523

Tegmark, M., \& Efstathiou, G. 1996, MNRAS, 281, 1297

Tristram, M., Macias-Perez, J. F., Renault, C., \& Santos, D. 2005, MNRAS, 358,833

Zaldarriaga, M., \& Seljak, U. 1997, Phys. Rev. D, 55, 1830

Zaldarriaga, M., Spergel, D. N., \& Seljak, U. 1997, ApJ, 488, 1 